\title{
Unrecognized bony Bankart lesion accompanying a dislocated four-part proximal humerus fracture before surgery:
} a case report

\author{
Seungjin Lee, Daehun Shin, Yoonsuk Hyun \\ Department of Orthopedic Surgery, Kangdong Sacred Heart Hospital, Seoul, Korea
}

\begin{abstract}
Proximal humerus fractures are the third most common fractures, totaling $4 \%$ to $5 \%$ of all fractures. Here, we present the case of a 39 -yearold man with a dislocated four-part fracture of the proximal humerus with a huge bony Bankart lesion. Preoperatively, the bony Bankart lesion of the glenoid was not visualized on computed tomography scans or magnetic resonance imaging because the fracture of the proximal humerus was comminuted, displaced, and complex. It was planned for only the humerus fracture to be treated by open reduction and internal fixation using a locking plate. However, a fractured fragment remained under the scapula after reduction of the dislocated humeral head. This was mistaken for a dislocated bone fragment of the greater tuberosity and repositioning was attempted. After failure, visual confirmation showed that the bone fragment was a piece of the glenoid. After reduction and fixation of this glenoid part with suture anchors, we acquired a well-reduced fluoroscopic image. Given this case of complex proximal humerus fracture, a glenoid fracture such as a bony Bankart lesion should be considered preoperatively and intraoperatively in such cases.
\end{abstract}

Keywords: Humerus; Humerus fracture; Shoulder dislocation; Bankart lesions

Proximal humerus fractures are the third most common fractures, totaling $4 \%$ to $5 \%$ of all fractures. Most of these fractures occur in elderly patients with osteoporosis due to minor trauma. However, one-fourth of these fractures occur in young people who experience high-energy accidents. Combined injury of a complex proximal humerus fracture with a glenoid fracture is rare. To our knowledge, few reports about this condition are available in the literature; there is a previous case series of two complex proximal humerus fractures concomitant with a bony Bankart lesion treated with hemiarthroplasty and glenoid rim fixation [1]. Also, a case series of 26 patients with proximal hu- merus fracture and glenoid fractures treated with reverse total shoulder arthroplasty and bone grating was reported [2]. Shoulder dislocations occur in two-thirds of all large anterior glenoid rim fractures, complicating treatment [3]. We describe a case of dislocated four-part proximal humerus fracture with an intraarticular glenoid fracture (bony Bankart lesion), which was overlooked preoperatively. This case was treated with simultaneous open reduction and internal fixation of the proximal humeral fracture and glenoid fracture. If a bony Bankart lesion is neglected and not properly fixed, it can be difficult to obtain stable reduction, the dislocation might recur after surgery. Therefore, we

Received: October 28, $2021 \quad$ Revised: November 20, $2021 \quad$ Accepted: November 22, 2021

Correspondence to: Yoonsuk Hyun

Department of Orthopedic Surgery, Kangdong Sacred Heart Hospital, 150 Seongan-ro, Gangdong-gu, Seoul 05355, Korea

Tel: +82-2-2224-2230, Fax: +82-2-489-4391, E-mail: yshyun72@gmail.com, ORCID: https://orcid.org/0000-0003-3826-2527

Financial support: None.

Conflict of interest: None.

Copyright@ 2022 Korean Shoulder and Elbow Society.

This is an Open Access article distributed under the terms of the Creative Commons Attribution Non-Commercial License (http://creativecommons.org/licenses/by-nc/4.0/) which permits unrestricted non-commercial use, distribution, and reproduction in any medium, provided the original work is properly cited. 
recommend suspecting a glenoid fracture preoperatively in the presence of a complex proximal humerus fracture with dislocation.

\section{CASE REPORT}

Owing to the retrospective design of this case study including no more than three cases, approval of the Institutional Review Board was waived. The Institutional Review Board of Kangdong Sacred Heart Hospital approved the waiver of informed consent for this case report, but the authors obtained the informed consent from the patient.

A 39-year-old man slipped while riding an electric scooter and injured himself. He was transferred to the emergency room of our hospital by ambulance. On plain radiographs and computed tomography (CT) scans, there was a four-part proximal humerus fracture with anterior dislocation of the humeral head fragment of the right shoulder (Fig. 1). After confirming that there was no neurovascular abnormality in the right upper extremity, manual reduction of the dislocation was attempted but was unsuccessful. For evaluation of soft-tissue injury around the shoulder, CT scans with three-dimensional reconstruction and magnetic resonance imaging (MRI) were performed. On imaging, scattered fractured fragments of the proximal humerus and dislocated humeral head impeded precise evaluation of the glenoid bone, and no bony Bankart lesion was detected (Fig. 2). Although there is a risk of avascular necrosis after osteosynthesis in dislocated fourpart proximal humerus fracture, open reduction surgery with in- ternal fixation using a locking plate was performed considering the age and activity level of the patient.

Under satisfactory general anesthesia, the patient was placed on the operating table in the beach chair position. Routine skin preparation and draping were completed in a sterile manner. With the deltopectoral approach, the skin was incised to the fracture site. The dislocated humerus head was easily detected and repositioned by pulling with a towel clip. When the pulling force was relaxed, the humeral head showed a tendency to dislocate again (Fig. 3A). A single fractured bone fragment below the scapula was detected on the $\mathrm{C}$-arm fluoroscopic image and was as-
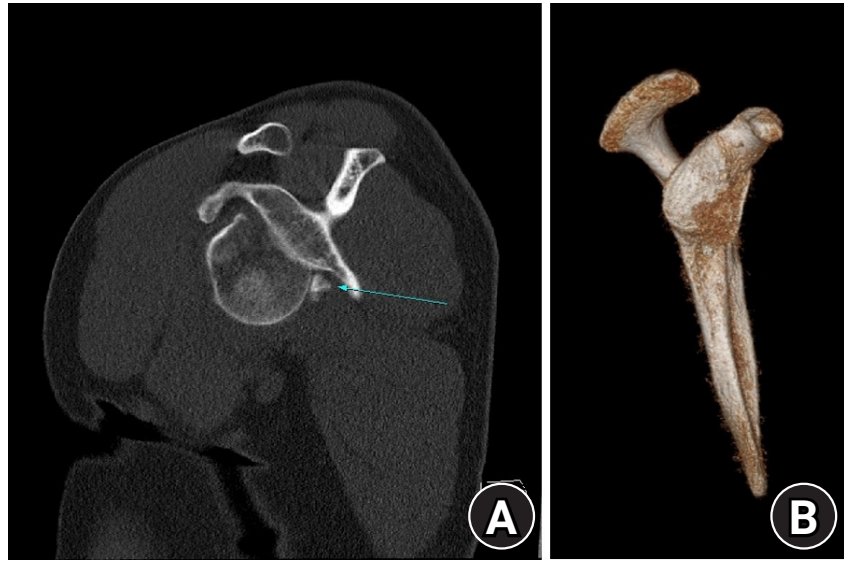

Fig. 2. Preoperative computed tomography $(\mathrm{CT})$ and magnetic resonance imaging findings. (A) CT sagittal view with arrow indicating the bony Bankart fragment. (B) The glenoid lesion could not be evaluated precisely on three-dimensional reconstruction CT images.
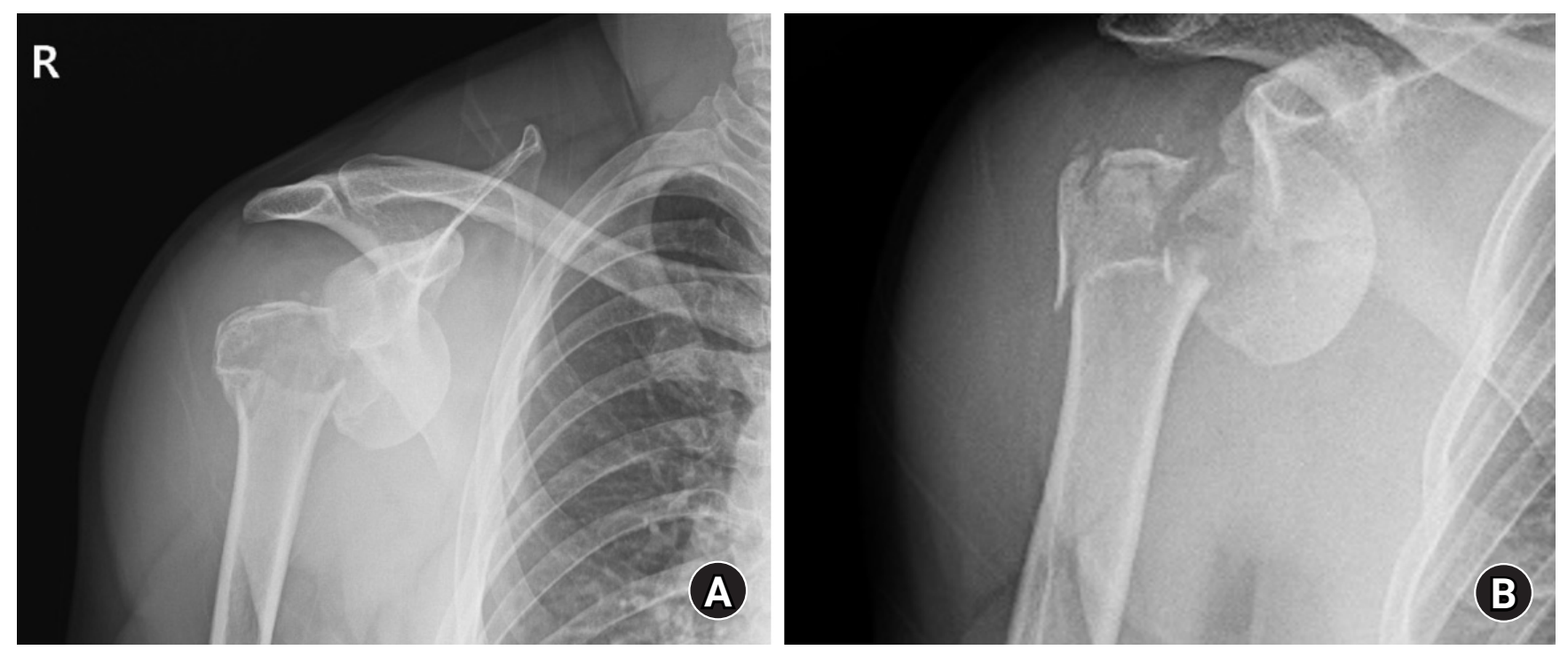

Fig. 1. Preoperative plain radiographic findings revealing a dislocated four-part fracture of the proximal humerus: (A) shoulder anteroposterior view and (B) true shoulder anteroposterior view. 

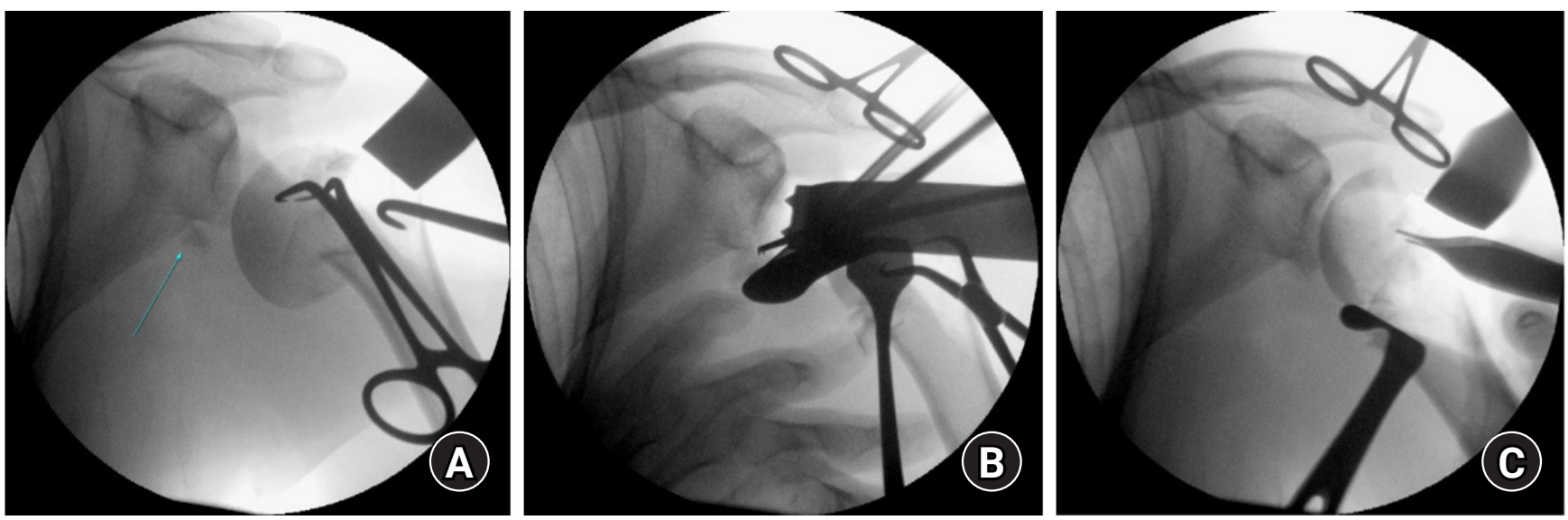

Fig. 3. Intraoperative findings on fluoroscopic images. (A) Unstable reduction of the humeral head fragment with arrow indicating the bony Bankart fragment. (B) The fractured fragment under the scapula was pulled several times with straight Kelly forceps under fluoroscopic guidance but failed to move. (C) Stable reduction was obtained after fixation of the bony Bankart lesion.

sumed to be a fractured fragment that did not return to its original position. This fragment was pulled several times with straight Kelly forceps under fluoroscopic guidance but failed to move (Fig. 3B). After the glenoid surface of the scapula could be observed between the displaced fragments, the fragment was identified as a piece of the glenoid, a bony Bankart lesion. There was no need to split the subscapularis for access to the bony Bankart lesion because the lesser tuberosity had been fractured and separated from the head fragment. Therefore, access to the bony Bankart lesion was possible through the space obtained by retracting the proximal humerus fragments without damaging the subscapularis. The bony Bankart lesion was fixed with two Suturefix Ultra suture anchors (Smith \& Nephew, London, England) using a suture bridge technique. Stable reduction of the humeral head was achieved (Fig. 3C). For prevention of humeral head fragment collapse, we placed an allogenic tricortical iliac bone-block (Community Tissue Services, Kettering, OH, USA) under the head fragment, and final fixation was performed using a locking plate.

We confirmed that the reduced fractures in the humerus and glenoid were well maintained on CT scans at 3 days after surgery (Fig. 4). Passive range of motion within tolerable limits began 4 days after surgery to prevent stiffness, and the patient was discharged from the hospital 6 days after surgery. At 4 months after surgery, the patient had achieved $170^{\circ}$ of forward elevation and almost complete union of the fracture (Fig. 5).

\section{DISCUSSION}

This study is a case report of a four-part fracture-dislocation of the proximal humerus with a bony Bankart lesion. The patient was treated for the first time with internal plate fixation for the humerus and glenoid rim by suture-anchors. According to a study on classification and incidence of proximal humerus fractures, four-part fracture- dislocations account for $1 \%$ of all proximal humerus fractures [4]. In another study by Iglesias-Rodríguez et al. [5], 36 (5.6\%) of 638 patients with proximal humerus fracture had glenohumeral joint dislocation. Furthermore, fractures of the proximal humerus are rarely accompanied by scapular glenoid fractures. Limited data exist in the literature concerning these complex fractures [6].

Since these fractures involve many displaced bony fragments, it is often difficult to identify whether a glenoid fracture is present on preoperative images. Therefore, in the case of a complex proximal humeral fracture, glenoid fracture should always be suspected, and the surgical strategy should be planned with this in mind. Meanwhile, the indications for fixation of glenoid fractures include severe and displaced fractures with an articular step-off of 3 to $10 \mathrm{~mm}$ as well as a fracture fragment involving more than $20 \%$ of the glenoid surface. Failing to address displaced glenoid fractures can lead to poor outcomes and instability. A higher incidence of recurrent instability or secondary osteoarthritis has been reported in patients with glenoid fractures associated with proximal humeral fracture [7].

In terms of the sequence of fixation for this injury, Ong et al. [8] proposed starting with the proximal humerus fracture. The glenohumeral joint can be exposed by splitting the subscapularis muscle, which is later re-sutured. A locking plate can be used for the humerus, with sutures in the tendinous cuff allowing the implant to parachute down to the humeral head, aiding reduction. We think it ultimately does not matters which is addressed firstthe humerus or glenoid-but the glenoid fracture was addressed first in this case through the space between displaced fractured 

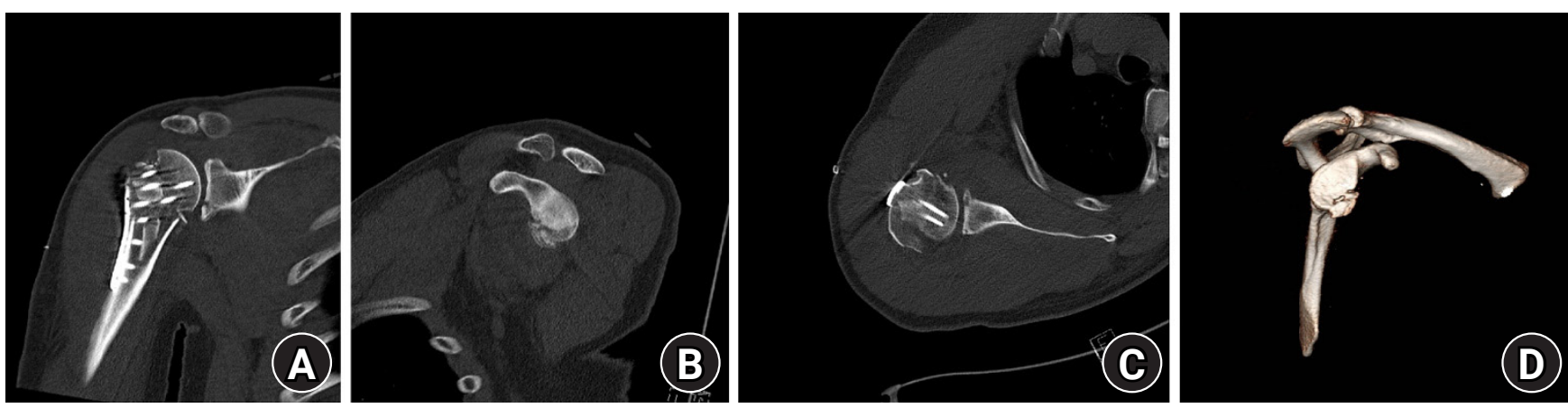

Fig. 4. Postoperative computed tomography (CT) scans showing a fixed bony Bankart lesion: (A) coronal view, (B) sagittal view, (C) axial view, and (D) three-dimensional reconstruction CT image.

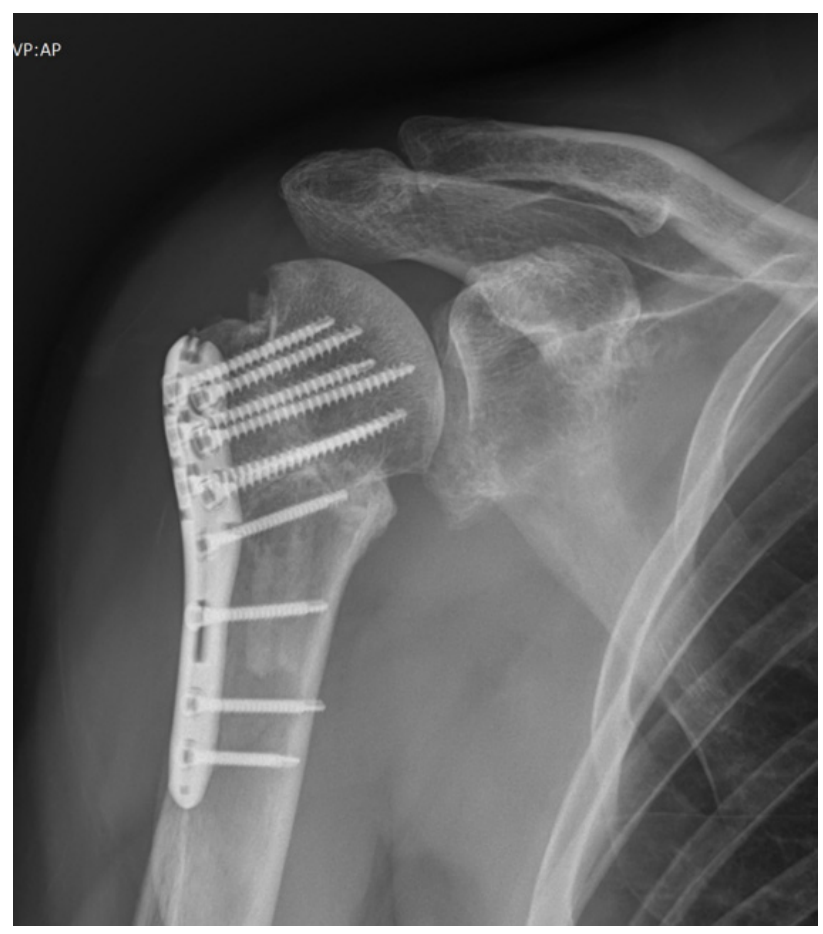

Fig. 5. Plain radiograph (true shoulder anteroposterior view) at 3 months after surgery shows bony union.

fragments of the humeral head.

At the 4-month follow-up examination, the patient showed $170^{\circ}$ of active forward elevation. A high probability of humeral head necrosis and instability must be considered in three-and four-part proximal humerus fractures with bony Bankart lesions [9]. Afterward, at least 18 months of outpatient follow-up is necessary to monitor for complications, including development of avascular necrosis in the humeral head [10].

In conclusion, we present a case of a patient with a dislocated four-part fracture of the proximal humerus and a bony Bankart lesion. Preoperatively, the bony Bankart lesion was missed on the CT scans and MRI. If we did not detect and manage this bony
Bankart lesion, we might have failed to obtain stable reduction of the glenohumeral dislocation. Given this case of complex proximal humerus fracture, it is necessary to suspect a glenoid fracture such as a bony Bankart lesion preoperatively and intraoperatively in such cases.

\section{ORCID}

Seungjin Lee

https://orcid.org/0000-0001-8951-0039

Daehun Shin

https://orcid.org/0000-0003-2280-9081

Yoonsuk Hyun

https://orcid.org/0000-0003-3826-2527

\section{REFERENCES}

1. Lahav A, Rogachefsky R, Toledano B, Meinhard BP. The management of anterior dislocation of the shoulder with proximal humerus fracture, bony Bankart lesion, and associated glenoid labrum avulsion: a report of 2 cases. Am J Orthop (Belle Mead NJ) 2003;32:349-52

2. Garofalo R, Brody F, Castagna A, Ceccarelli E, Krishnan SG. Reverse shoulder arthroplasty with glenoid bone grafting for anterior glenoid rim fracture associated with glenohumeral dislocation and proximal humerus fracture. Orthop Traumatol Surg Res 2016;102:989-94.

3. Oh JC, Kim HS, Lee GH, Kang HJ. Arthroscopic treatment of a one-fourth anteroinferior glenoid comminuted fracture-dislocation with concomitant three-part complex proximal humerus fracture in a middle-aged man: a case report. Arch Orthop Trauma Surg 2020;140:1901-7.

4. Court-Brown CM, Garg A, McQueen MM. The epidemiology of proximal humeral fractures. Acta Orthop Scand 2001;72:36571.

5. Iglesias-Rodríguez S, Domínguez-Prado DM, García-Reza A, et al. Epidemiology of proximal humerus fractures. J Orthop Surg 
Res 2021;16:402.

6. Maassen NH. Treatment of a 4-part proximal humeral fracture with a concomitant glenoid fracture in an elderly patient: a case report. JBJS Case Connect 2019;9:e13.

7. Attarde D, Patil A, Kamat N, Sancheti P, Shyam A. Anterior proximal humerus fracture dislocation with concomitant glenoid fracture: an operative challenge! A case report. Trauma 2021 May 17 [Epub]. https://doi.org/10.1177/14604086211019 528.

8. Ong JC, Awadallah MA, White AJ, Robinson PM. The 'floating humeral head', a case series of combined proximal humeral and glenoid fracture dislocations. J Orthop Orthop Surg 2020;1:1924.

9. Tamai K, Ishige N, Kuroda S, et al. Four-segment classification of proximal humeral fractures revisited: a multicenter study on 509 cases. J Shoulder Elbow Surg 2009;18:845-50.

10. Solberg BD, Moon CN, Franco DP, Paiement GD. Locked plating of 3- and 4-part proximal humerus fractures in older patients: the effect of initial fracture pattern on outcome. J Orthop Trauma 2009;23:113-9. 